\title{
miR-146a gene polymorphism rs2910164 and the risk of digestive tumors: A meta-analysis of 21 case-control studies
}

\author{
XIAOHUI XU ${ }^{1 *}$, XIAODONG YANG $^{1 *}$, GAN RU $^{1}$, YONG WU $^{1}$, SHUYU ZHANG $^{2}$, \\ CHUNGEN XING ${ }^{1}$, YONGYOU WU ${ }^{1}$ and JIANPING CAO ${ }^{2}$ \\ ${ }^{1}$ Department of General Surgery, The Second Affiliated Hospital of Soochow University, Suzhou 215004; \\ ${ }^{2}$ School of Radiation Medicine and Protection, Medical College of Soochow University, Suzhou 215123, P.R. China
}

Received August 23, 2013; Accepted October 15, 2013

DOI: $10.3892 / o r .2013 .2854$

\begin{abstract}
Digestive tumors have the highest incidence among all tumor types worldwide. miR-146a has been shown to play an important role in the development, apoptosis, invasion and metastasis of digestive tumors. Additionally, a miR-146a gene polymorphism has been associated with the risk of a variety of cancer types in the digestive system. Therefore, in order to investigate the correlation, a meta-analysis of reported data was conducted, for which we obtained 21 research studies concerning the association between the miR-146a gene polymorphism and digestive tumors. Odds ratio (OR) values and $95 \%$ confidence intervals $(95 \% \mathrm{CI})$ were used to assess this association. We found that the miR-146a polymorphism rs2910164 might significantly increase the susceptibility of digestive tumors, in particular for esophageal cancer and colorectal cancers. Furthermore, the miR-146a polymorphism might significantly increase the risk of digestive tumors in Asians. However, no obvious correlation between the polymorphism and the risk for digestive tumors was found in Caucasians.
\end{abstract}

\section{Introduction}

According to global cancer statistics, the morbidity and mortality rates of digestive tumors rank first in both male and female populations (1). The etiology and pathogenesis of diges-

Correspondence to: Professor Chungen Xing, Department of General Surgery, The Second Affiliated Hospital of Soochow University, No. 1055, Sanxian Road, Suzhou 215004, P.R. China

E-mail:cg_xing@aliyun.com

Dr Shuyu Zhang, School of Radiation Medicine and Protection, Medical College of Soochow University, No. 199, Renai Road, Suzhou 215123, P.R. China

E-mail: zhang.shuyu@hotmail.com

${ }^{*}$ Contributed equally

Key words: miR-146a, digestive tumor risk, polymorphism, meta-analysis tive tumors have a lot in common. To prevent digestive tumors, it is necessary to understand the related predisposing factors. Studies have shown that environmental factors, diet, intake of non-steroidal and anti-inflammatory drugs, and endogenous factors can significantly affect the individual susceptibility to digestive tumors $(2,3)$.

MicroRNAs (miRNAs) are a group of small non-coding RNAs that are 22 (18-25) nucleotides (nt) long. They have been found to be associated with a variety of diseases, including cancer. An increasing number of findings confirm that miRNAs play essential roles in the development, but also the diagnosis, treatment and prognosis of a variety of tumors. The value of using miRNAs as biomarkers for diagnosis and as target molecules for treatment of cancer is increasingly being recognized (4).

Numerous studies have shown that miR-146a is involved in the development, apoptosis, invasion and metastasis of digestive tumors; studies have reported that miR-146a is downregulated in gastric cancer or gastric cancer cells and that it regulates cell proliferation and apoptosis of gastric cancer cells (5). In addition, Vinci et al (6) studied the distribution of sequence variants of miR-146a in colorectal cancer and the effects of miRNA expression. He et al (7) reported that miR-146a modulated TGF- $\beta 1$-induced hepatic stellate cell proliferation by targeting SMAD4. Tomokuni et al (8) demonstrated that miR-146a inhibited the anticancer effect of IFN- $\alpha$ in hepatocellular cancer (HCC) cells, and that this effect was mediated by SMAD4. Another study found that the expression of miR-146a inhibited the invasive capacity of pancreatic cancer cells with concomitant downregulation of EGFR and the NF- $\mathrm{kB}$ regulatory kinase interleukin 1 receptor-associated kinase 1 (9). Therefore, miR-146a appears to play a crucial role in the properties of digestive tumors.

Studies have shown that single nucleotide polymorphisms (SNPs) in human miRNAs constitute one of the main forms of genetic variation in human genomic DNA sequences and that they might play central roles in the susceptibility to human disease. miRNA SNPs exhibit interindividual differences that are relevant to disease diagnosis, treatment and prognosis.

A number of recent studies have suggested that the miR-146a expression is deregulated in numerous solid tumors, and it has become evident that miR-146a might act as a tumor-suppressor $(5,10,11)$. The miR-146a rs2910164 G>C 
polymorphism is caused by the miR-146a leader sequence $\mathrm{G}: \mathrm{U}$ and $\mathrm{C}: \mathrm{U}$ base pair mismatching. Studies have shown that the miR-146a gene polymorphism rs2910164 is associated with the occurrence of a variety of cancer types, such as prostate, breast and cervical cancer (12-14). From 2008 to 2013, researchers have repeatedly reported associations between the miR-146a rs2910164 polymorphism and the risk for digestive tumors, but the results were mixed or even conflicting. Therefore, we performed a meta-analysis to derive a more precise estimation of the association between the miR-146a G/C SNP and the risks of developing cancer in the digestive system.

\section{Materials and methods}

Screening and identification of relevant studies. Identification and eligibility of relevant studies was performed using the search terms 'miR/microRNA-146a', 'digestive cancer', 'biliary cancer', 'hepatocellular cancer', 'esophageal squamous cell carcinoma', 'gastric cancer', 'colorectal cancer', 'pancreatic cancer', 'rs2910164', 'genotype', 'polymorphism' and 'variant' in the PubMed, Ovid and Embase databases and in the Cochrane Library (last search update: 17 April, 2013). The search was limited to English language articles and only published studies with full-text articles were included. We evaluated potentially relevant publications by manually examining their title and abstract.

Inclusion and exclusion criteria. Inclusion criteria were: i) assessment of miR-146a rs2910164 polymorphism and the risk of suffering from digestive cancer; ii) a separate casecontrol study on humans; iii) statistically sound genotype data with odds ratio (OR) values and $95 \%$ confidence intervals $(\mathrm{CI})$; iv) full-text search. Exclusion criteria were: i) lack of controls in the studies; ii) repetition of previous results; iii) summary, comment, review and editorial articles; iv) a focus on benign tumors of the digestive tract.

Data extraction and study characteristics. Two researchers (Xiaohui Xu and Xiaodong Yang) independently extracted all data that met the above inclusion criteria and the existing differences in the resulting datasets were resolved by team discussions. From each study, the following information was extracted: last name of the first author, year of publication, ethnicity, tumor type, source of research, research methods, the number of cases and controls, the number of various genotypes of cases and controls. If a study did not provide complete data, we sent requests for this information to the corresponding author. A total of 21 eligible studies, comprising 10,318 cases and 12,478 controls met the inclusion criteria (Table I). The studies were published in the period from 2008 to 2013, and all were case-control studies. The case groups were only suffering from one type of cancer (gastrointestinal), while the control groups did not present with any tumor. From these 21 studies, 16 involved individuals of Asian ethnicity and 5 of Caucasian. In addition, the 21 studies used different detection methods, with 14 studies using the traditional method of polymerase chain reaction-restriction fragment length polymorphism (PCR-RFLP), 1 using the PCR confronting two-pair primer (PCR-CTPP) method, 4 using the TaqMan-polymerase chain reaction (TaqMan-PCR) and another 2 using a SNP assay. All the control groups were selected from a healthy population and the age and gender were consistent with cases. All the above data complied with the Hardy-Weinberg equilibrium (HWE) principles (Table I).

Statistical analysis. According to the case and control genotype frequencies, the correlation between the miR-146a rs2910164 polymorphism and the risk of gastrointestinal cancer was assessed via OR values with $95 \%$ CI. In addition, we analyzed whether tumor type and ethnicity may affect the relationship between the miR-146a rs2910164 polymorphism and the risk of gastrointestinal cancer. Statistical analysis was performed on OR values (with 95\% CI) for 5 distinct genotypic comparisons: the allelic one (G vs. C), comparison to the dominant genetic model ( $\mathrm{GC}+\mathrm{GG}$ vs. $\mathrm{CC})$, comparison to the recessive genetic model ( $\mathrm{GG}$ vs. $\mathrm{GC}+\mathrm{CC}$ ), the homozygote (GG vs. CC) and the heterozygote comparison (GC vs. CC). The Chi-square-based Q statistic was used to assess heterogeneity between studies, with a p-value $\left(\mathrm{P}_{\text {heterogeneity }}\right)<0.05$ considered to indicate statistically significant heterogeneity between studies. The $\mathrm{I}^{2}$ index, expressed as a percentage, quantified the degree of heterogeneity throughout the study, with $\mathrm{I}^{2}$ values of 25,50 and $75 \%$ referring to low, medium and high heterogeneity, respectively. Funnel plots were used to assess the publication bias. When the effects were assumed to be homogenous, the fixed-effects model was used (MantelHaenszel method). If inter-study heterogeneity was detected, the random-effects model was applied (DerSimonian and Laird method) (15). All data analyses were performed using the software Stata 11.0 and all the p-values are derived twosided tests.

\section{Results}

Study characteristics. A total of 545 articles relevant to the used search terms were identified, and only 32 studies concerned the association between digestive cancer and the miR-146a rs2910164 polymorphism. According to the inclusion and exclusion criteria described in Materials and methods, 21 publications (16-36) were included in the final meta-analysis, 8 using population-based controls and 13 using hospital-based controls (Fig. 1). From the 21 publications, 2 concerned esophageal, 4 colorectal, 6 gastric and 6 hepatocellular cancer. The main characteristics of the studies included in the meta-analysis are summarized in Table I.

Overall analyses. The overall analysis of all cases revealed a statistically significant positive association between the miR-146a rs2910164 polymorphism and the risk of developing digestive tumors (Fig. 2).

Allele G vs. C: OR=1.08, 95\% CI: 1.04-1.12, $\mathrm{P}_{\text {heterogeneity }}=0.001$ (Fig. 2A). The results suggested that individuals with the $\mathrm{G}$ allele were more susceptible to digestive cancer than those with the $\mathrm{C}$ allele.

Dominant genetic model GC + GG vs. CC: OR=1.11, 95\% CI: 1.04-1.18, $\mathrm{P}_{\text {heterogeneity }}<0.001$ (Fig. 2B). The results suggested that individuals following the dominant genetic model GC + GG may show an increased susceptibility to digestive cancer. 


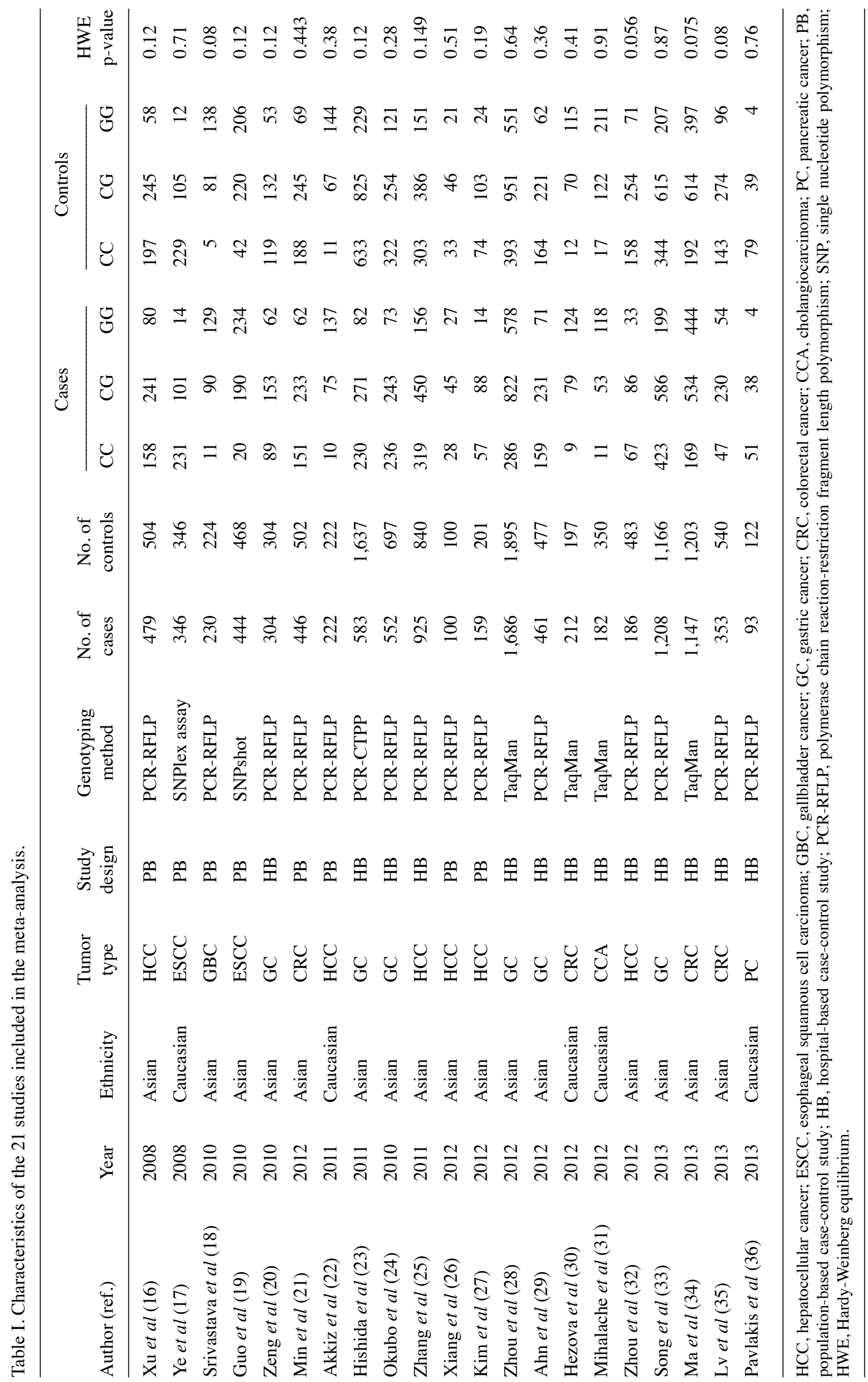




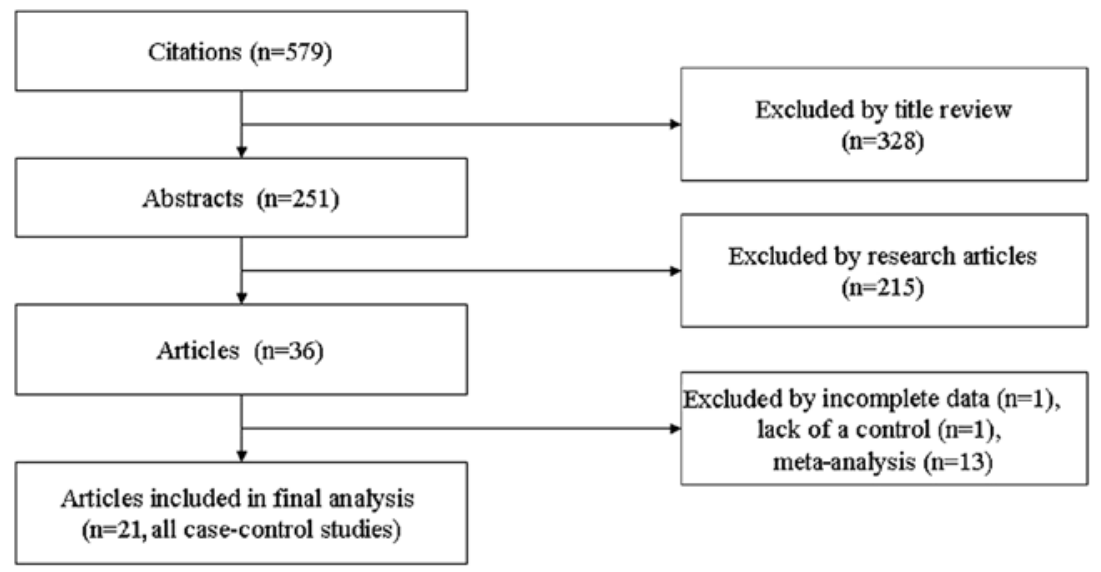

Figure 1. Flowchart of the selection of published studies.

A

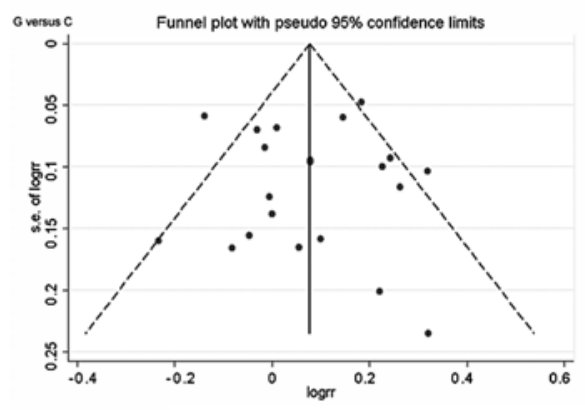

B

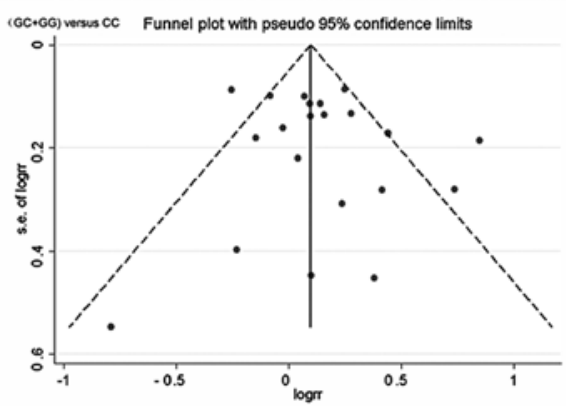

C

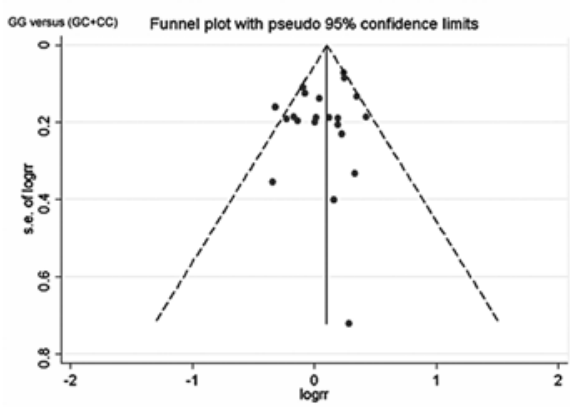

D $\quad$ E
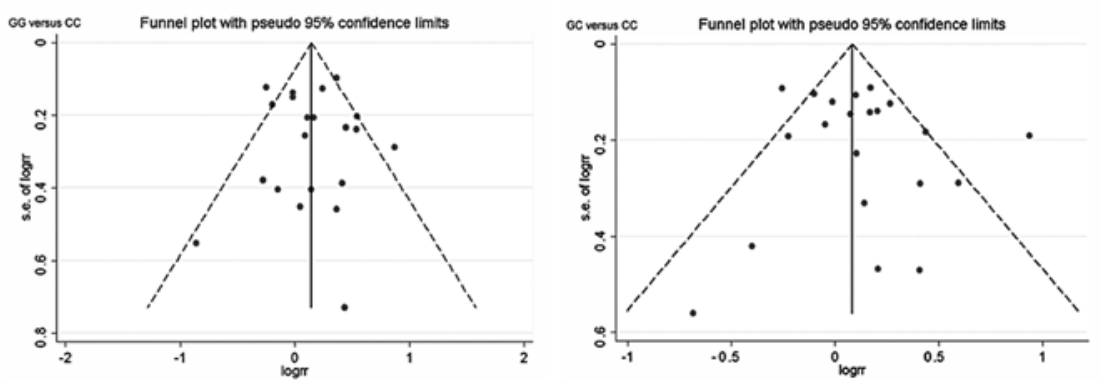

Figure 2. Forest plot for the association between the miR-146a rs2910164 polymorphism and digestive cancer risk in the populations. (A) G vs. C; (B) GC + GG vs. CC; (C) GG vs. GC + CC; (D) GG vs. CC; (E) GC vs. CC.

Recessive genetic model GG vs. $\mathrm{CC}+\mathrm{GC}$ : $\mathrm{OR}=1.11$, 95\% CI: $1.04-1.18, \mathrm{P}_{\text {heterogeneity }}=0.020$ (Fig. $2 \mathrm{C}$ ). The results suggested that compared to $\mathrm{CC}+\mathrm{GC}$, individuals following the recessive genetic model GG were more susceptible to digestive cancer.

Homozygous GG vs. CC: OR=1.16, 95\% CI: 1.06-1.26, $\mathrm{P}_{\text {heterogeneity }}=0.002$ (Fig. 2D). The results suggested that individuals following the homozygous GG model showed no significant difference compared to individuals with the CC allele with regards to developing digestive cancer.

Heterozygous GC vs. CC: OR=1.09, 95\% CI: 1.02-1.16, $\mathrm{P}_{\text {heterogeneity }}<0.001$ (Fig. 2E). The results suggested that individuals following the heterozygous model GC were more susceptible to digestive cancer than those with the $\mathrm{CC}$ allele.
Furthermore, in the stratified analysis exploring the contributions from the type of digestive cancer (Table II), significantly increased risks for esophageal squamous cell carcinoma were found [(for G vs. C: 1.23 (1.04-1.44), $\mathrm{P}_{\text {heterogeneity }}=0.054$; for GG vs. CC+GC: 1.39 (1.09-1.78), $\mathrm{P}_{\text {heterogeneity }}=0.655$; for GG vs. CC: 1.88 (1.19-2.97), $\left.\left.\mathrm{P}_{\text {heterogeneity }}=0.145\right)\right]$, as well as risks for colorectal cancer [(for G vs. C: 1.15 (1.06-1.25), $\mathrm{P}_{\text {heterogeneity }}=0.697$; for $(\mathrm{GC}+\mathrm{GG})$ vs. $\mathrm{CC}: 1.31$ (1.12-1.52), $\mathrm{P}_{\text {heterogeneity }}=0.005$, for $\mathrm{GG}$ vs. $\mathrm{CC}+\mathrm{GC}: 1.14(1.00-1.31), \mathrm{P}_{\text {heterogeneity }}=0.16$; for $\mathrm{GG}$ vs. CC: 1.30 (1.08-1.57), $\mathrm{P}_{\text {heterogeneity }}=0.587$, for GC vs. CC: 1.28 $\left.\left.(1.09-1.50), \mathrm{P}_{\text {heterogeneity }}<0.001\right)\right]$, but not for biliary, hepatocellular, gastric and pancreatic cancer.

However, in the subgroup analysis where ethnicity was analyzed, significantly increased risks were found for Asians 
Table II. Subgroup analysis of the association between the miR-146a rs2910164 polymorphism and the risk of digestive cancer.

\begin{tabular}{|c|c|c|c|c|c|}
\hline Comparison & Subgroup & No. ${ }^{a}$ & OR $(95 \% \mathrm{CI})$ & $\mathrm{I}^{2}(\%)$ & P-value ${ }^{b}$ \\
\hline \multirow[t]{10}{*}{ G vs. C } & Ethnicity & & & & \\
\hline & Asian & 16 & $1.08(1.04-1.13)$ & 65.1 & $<0.001$ \\
\hline & Caucasian & 5 & $1.05(0.91-1.21)$ & 0.0 & 0.701 \\
\hline & Digestive cancer type & & & & \\
\hline & Biliary cancer & 2 & $0.94(0.75-1.17)$ & & \\
\hline & Hepatocellular cancer & 6 & $1.06(0.97-1.16)$ & 23.4 & 0.258 \\
\hline & Esophageal squamous cell carcinoma & 2 & $1.23(1.04-1.44)$ & 70.8 & 0.054 \\
\hline & Gastric cancer & 6 & $1.05(0.99-1.11)$ & 78.6 & $<0.001$ \\
\hline & Colorectal cancer & 4 & $1.15(1.06-1.25)$ & 0.0 & 0.697 \\
\hline & Pancreatic cancer & 1 & $1.38(0.87-2.18)$ & & \\
\hline \multirow[t]{10}{*}{$\mathrm{GC}+\mathrm{GG}$ vs. $\mathrm{CC}$} & Ethnicity & & & & \\
\hline & Asian & 16 & $1.11(1.04-1.18)$ & 72.5 & $<0.001$ \\
\hline & Caucasian & 5 & $1.08(0.85-1.37)$ & 0.0 & 0.576 \\
\hline & Digestive cancer type & & & & \\
\hline & Biliary cancer & 2 & $0.65(0.35-1.23)$ & & \\
\hline & Hepatocellular cancer & 6 & $1.10(0.97-1.26)$ & 0.0 & 0.549 \\
\hline & Esophageal squamous cell carcinoma & 2 & $1.19(0.91-1.56)$ & 82.1 & 0.018 \\
\hline & Gastric cancer & 6 & $1.04(0.96-1.14)$ & 79.7 & $<0.001$ \\
\hline & Colorectal cancer & 4 & $1.31(1.12-1.52)$ & 76.5 & 0.005 \\
\hline & Pancreatic cancer & 1 & $1.51(0.87-2.63)$ & & \\
\hline \multirow[t]{10}{*}{ GG vs. $\mathrm{CC}+\mathrm{GC}$} & Ethnicity & & & & \\
\hline & Asian & 16 & $1.12(1.04-1.19)$ & 54.6 & 0.005 \\
\hline & Caucasian & 5 & $1.04(0.84-1.29)$ & 0.0 & 0.792 \\
\hline & Digestive cancer type & & & & \\
\hline & Biliary cancer & 2 & $0.98(0.76-1.28)$ & & \\
\hline & Hepatocellular cancer & 6 & $1.06(0.90-1.24)$ & 40.8 & 0.133 \\
\hline & Esophageal squamous cell carcinoma & 2 & $1.39(1.09-1.78)$ & 0.0 & 0.655 \\
\hline & Gastric cancer & 6 & $1.09(0.99-1.20)$ & 64.5 & 0.015 \\
\hline & Colorectal cancer & 4 & $1.14(1.00-1.31)$ & 42 & 0.16 \\
\hline & Pancreatic cancer & 1 & $1.33(0.32-5.45)$ & & \\
\hline \multirow[t]{10}{*}{ GG vs. CC } & Ethnicity & & & & \\
\hline & Asian & 16 & $1.16(1.06-1.26)$ & 64.1 & $<0.001$ \\
\hline & Caucasian & 5 & $1.12(0.75-1.68)$ & 0.0 & 0.92 \\
\hline & Digestive cancer type & & & & \\
\hline & Biliary cancer & 2 & $0.68(0.36-1.28)$ & & \\
\hline & Hepatocellular cancer & 6 & $1.14(0.95-1.38)$ & 28.8 & 0.219 \\
\hline & Esophageal squamous cell carcinoma & 2 & $1.88(1.19-2.97)$ & 53 & 0.145 \\
\hline & Gastric cancer & 6 & $1.10(0.98-1.23)$ & 76.3 & 0.001 \\
\hline & Colorectal cancer & 4 & $1.30(1.08-1.57)$ & 0.0 & 0.587 \\
\hline & Pancreatic cancer & 1 & $1.55(0.37-6.47)$ & & \\
\hline \multirow[t]{10}{*}{ GC vs. CC } & Ethnicity & & & & \\
\hline & Asian & 16 & $1.09(1.02-1.17)$ & 71.6 & $<0.001$ \\
\hline & Caucasian & 5 & $1.06(0.83-1.36)$ & 0.0 & 0.446 \\
\hline & Digestive cancer type & & & & \\
\hline & Biliary cancer & 2 & $0.61(0.31-1.17)$ & & \\
\hline & Hepatocellular cancer & 6 & $1.09(0.95-1.25)$ & 0.0 & 0.629 \\
\hline & Esophageal squamous cell carcinoma & 2 & $1.13(0.85-1.50)$ & 73 & 0.054 \\
\hline & Gastric cancer & 6 & $1.03(0.94-1.13)$ & 77.4 & $<0.001$ \\
\hline & Colorectal cancer & 4 & $1.28(1.09-1.50)$ & 83.5 & $<0.001$ \\
\hline & Pancreatic cancer & 1 & $1.51(0.85-2.67)$ & & \\
\hline
\end{tabular}

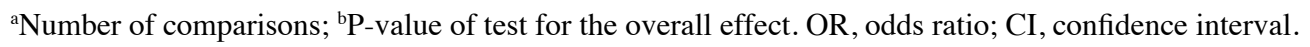


A

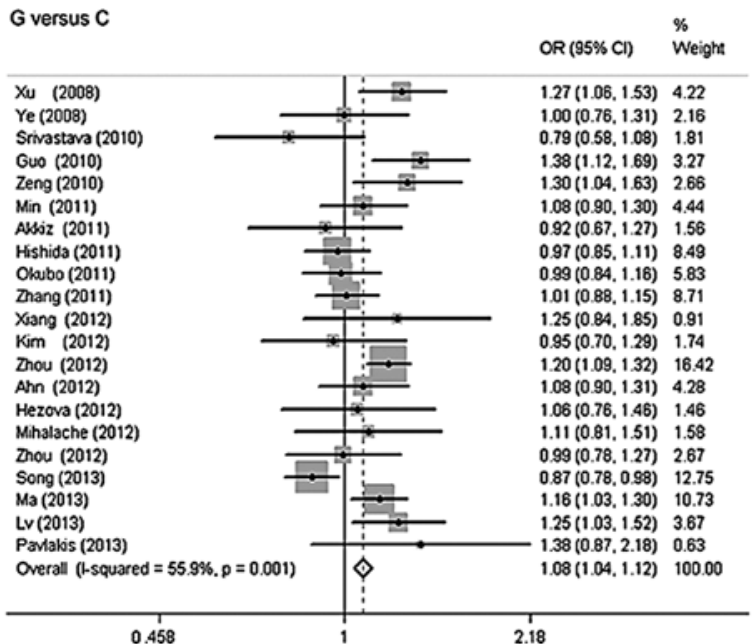

C

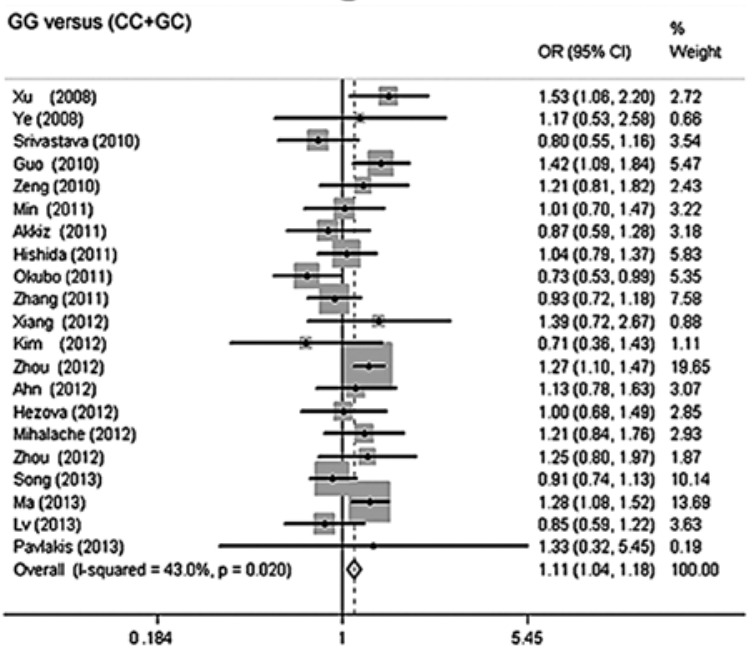

B

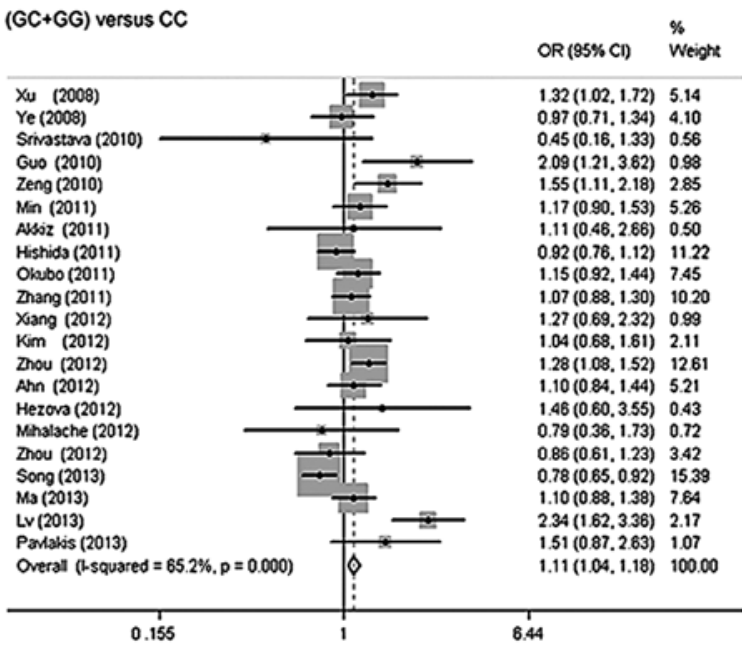

D

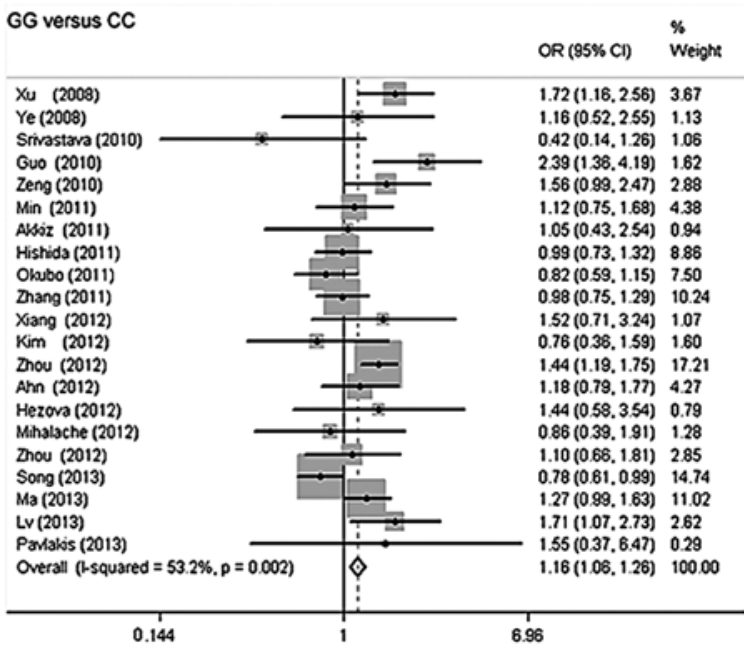

$E$

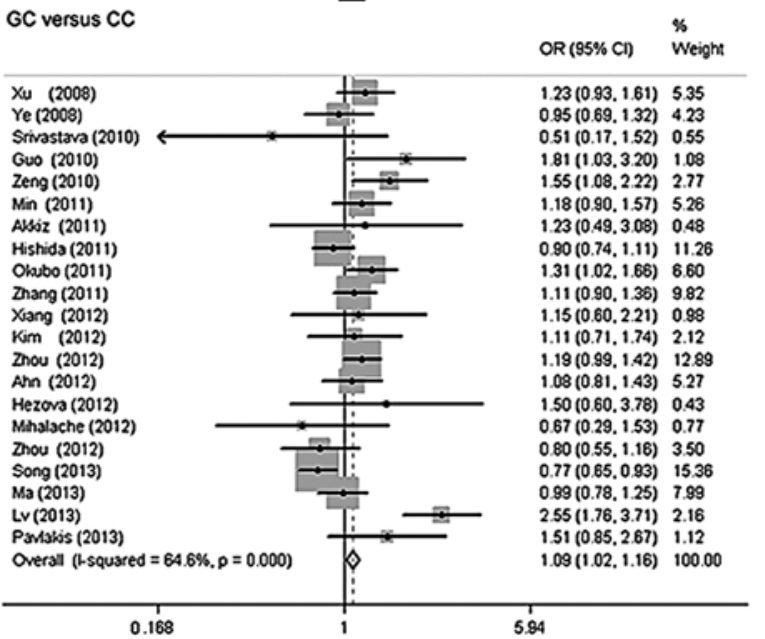

Figure 3. Funnel plot for all studies included in the meta-analysis studying associations of the miR-146a rs2910164 polymorphism with digestive cancer risk in the populations. (A) G vs. C; (B) GC + GG vs. CC; (C) GG vs. GC + CC; (D) GG vs. CC; (E) GC vs. CC.

[(for G vs. C: 1.08 (1.04-1.13), $\mathrm{P}_{\text {heterogeneity }}<0.001$; for $\mathrm{GC}+\mathrm{GG}$ vs. CC: 1.11 (1.04-1.18), $\mathrm{P}_{\text {heterogeneity }}<0.001$; for GG vs. $\mathrm{CC}+\mathrm{GC}$ : 1.12 (1.04-1.19), $\mathrm{P}_{\text {heterogeneity }}=0.005$; for GG vs. CC: $1.16(1.06-$
1.26), $\mathrm{P}_{\text {heterogeneity }}<0.001$; for GC vs. CC: 1.09 (1.02-1.17), $\left.\left.\mathrm{P}_{\text {heterogeneity }}<0.001\right)\right]$. No significant risk was found to be associated with any of the genetic models for Caucasians (Table II). 
Statistical sensitivity. Data from one study were omitted and the rest was analyzed, and the pooled RRs were similar with the overall pooled RRs (data not shown), supporting the robustness of our results.

Publication bias. Begg's funnel plot and Egger's test were used to assess the publication bias of the included studies. However, the results from all the comparisons and from Egger's test showed no evidence for a publication bias (Fig. 3): $\mathrm{P}=0.354$ for $\mathrm{G}$ vs. $\mathrm{C}, \mathrm{P}=0.912$ for $\mathrm{GC}+\mathrm{GG}$ vs. $\mathrm{CC}, \mathrm{P}=0.352$ for $\mathrm{GG}$ vs. $\mathrm{CC}, \mathrm{P}=0.795$ for $\mathrm{GC}$ vs. $\mathrm{CC}$ (except $\mathrm{P}=0.045<0.1$ for GG vs. $C C+\mathrm{GC}$ ).

\section{Discussion}

In the present study, we conducted a comprehensive statistical analysis of the relationship between the miR-146a polymorphism rs2910164 and digestive tumors. The results of the stratified analysis are the following: allele $\mathrm{G}$ vs. $\mathrm{C}(\mathrm{OR}=1.08$, 95\% CI: 1.04-1.12), dominant genetic model GC + GG vs. CC comparison $(\mathrm{OR}=1.11,95 \% \mathrm{CI}: 1.04-1.18)$, recessive genetic model GG vs. $\mathrm{CC}+\mathrm{GC}$ comparison $(\mathrm{OR}=1.11,95 \% \mathrm{CI}$ : 1.04-1.18), homozygous GG vs. $\mathrm{CC}$ comparison $(\mathrm{OR}=1.11$, 95\% CI: 1.04-1.18), heterozygous GC vs. CC comparison $(\mathrm{OR}=1.09,95 \% \mathrm{CI}: 1.02-1.16)$. We found that the miR-146a polymorphism rs2910164 might significantly increase the susceptibility to digestive tumors, especially for esophageal and colorectal cancer. In addition, the miR-146a polymorphism might significantly increase the risk for developing digestive tumors in Asian individuals, while for Caucasians, no obvious correlation between the polymorphism and the risk for digestive tumors was found.

miRNAs are small single-stranded regulatory RNAs the abnormal expression of which has been associated with the susceptibility to many human diseases, including cancer in the lung, prostate and bladder, cervical squamous cell carcinoma (37-40), autoimmune diseases such as systemic lupus erythematosus, Sjogren's syndrome and lupus nephritis (41-43), as well as cardiovascular diseases such as heart disease, heart failure and myocardial infarction (44-46).

The miR-146a polymorphism rs2910164 is associated with the susceptibility to a variety of tumors. Jazdzewski et al (47) found a significantly different distribution of genotypes among patients with papillary thyroid carcinomas as compared to normal subjects, with the GC genotype being associated with an increased risk of papillary thyroid carcinoma. Another study indicated that miR-146a might be involved in the pathogenesis of malignant melanoma, and individuals with the CG genotype showed an increased risk of developing malignant melanoma (48). Orsós et al (49) found that the pre-miR/146a $\mathrm{C}$ allele might contribute to an increased susceptibility to head and neck cancer. Therefore, the miR-146a polymorphism rs2910164 appears to be associated with the risk of developing cancer in a cancer type-specific manner.

The association between miR-146a polymorphisms and susceptibility to digestive tumors has attracted increased research in recent years. However, numerous studies on the topic were characterized by small sample size and thus, might not possess sufficient statistical power to detect effects of small magnitude or might have generated a fluctuated risk estimate. Moreover, conclusions from all these studies have not been uniform, and have even been contradicting for different types of tumors. Therefore, it is necessary to collect previously-generated research data and obtain a large number of samples to get reliable results.

Nevertheless, the present meta-analysis had a number of limitations. First, our study only concerned the analysis of unvaried factors. Second, the population characteristics of the experimental and the control groups were not uniform. Age, gender, HBV and potentially, other features, might have affected the reliability of the results. Third, the present study included only Caucasian and Asian populations; the absence of other ethnicities in the sample considerably reduces the universal validity of the results. Furthermore, unconsidered non-neoplastic disease factors might have impacted on the conclusions. Therefore, a more precise analysis might need to be performed.

In conclusion, our analysis demonstrated that there is an apparent association between the miR-146a polymorphism rs2910164 and digestive cancer. However, the association is inconsistent with regards to susceptibility to different types of gastrointestinal cancer. Therefore, it is necessary to collect large samples of data, perform stratified analyses and gather data from additional ethnicities to clarify the association between the miR-146a G/C rs2910164 polymorphism and the susceptibility to digestive cancer.

\section{Acknowledgements}

The present study was partially supported by the National Natural Science Foundation of China (grant nos. 81172348, 81102078 and 81172597), Health Research Projects in Jiangsu Province (H201313) and the Priority Academic Program Development of Jiangsu Higher Education Institutions (PAPD).

\section{References}

1. Jemal A, Bray F, Center MM, et al: Global cancer statistics. CA Cancer J Clin 61: 69-90, 2011.

2. Giovannucci E, Egan KM, Hunter DJ, et al: Aspirin and the risk of colorectal cancer in women. N Engl J Med 333: 609-614, 1995.

3. Landi D, Moreno V, Guino E, et al: Polymorphisms affecting micro-RNA regulation and associated with the risk of dietaryrelated cancers: a review from the literature and new evidence for a functional role of rs17281995 (CD86) and rs1051690 (INSR), previously associated with colorectal cancer. Mutat Res 717: 109-115, 2011.

4. Xu X, Yang X, Xing C, et al: miRNA: the nemesis of gastric cancer (Review). Oncol Lett 6: 631-641, 2013.

5. Hou Z, Xie L, Yu L, et al: MicroRNA-146a is down-regulated in gastric cancer and regulates cell proliferation and apoptosis. Med Oncol 29: 886-892, 2012.

6. Vinci S, Gelmini S, Mancini I, et al: Genetic and epigenetic factors in regulation of microRNA in colorectal cancers. Methods 59: 138-146, 2013.

7. He Y, Huang C, Sun X, et al: MicroRNA-146a modulates TGF-betal-induced hepatic stellate cell proliferation by targeting SMAD4. Cell Signal 24: 1923-1930, 2012.

8. Tomokuni A, Eguchi H, Tomimaru Y, et al: miR-146a suppresses the sensitivity to interferon- $\alpha$ in hepatocellular carcinoma cells. Biochem Biophys Res Commun 414: 675-680, 2011.

9. Li Y, Vandenboom TG II, Wang Z, et al: miR-146a suppresses invasion of pancreatic cancer cells. Cancer Res 70: 1486-1495, 2010.

10. Wang X, Tang S, Le SY, et al: Aberrant expression of oncogenic and tumor-suppressive microRNAs in cervical cancer is required for cancer cell growth. PLoS One 3: e2557, 2008. 
11. Yu J, Li A, Hong SM, et al: MicroRNA alterations of pancreatic intraepithelial neoplasias. Clin Cancer Res 18: 981-992, 2012.

12. Xu B, Wang N, Wang X, et al: MiR-146a suppresses tumor growth and progression by targeting EGFR pathway and in a p-ERK-dependent manner in castration-resistant prostate cancer. Prostate 72: 1171-1178, 2012.

13. Lian H, Wang L and Zhang J: Increased risk of breast cancer associated with CC genotype of $\mathrm{Hsa}$ - miR-146a Rs2910164 polymorphism in Europeans. PLoS One 7: e31615, 2012.

14. Yue C, Wang M, Ding B, et al: Polymorphism of the premiR-146a is associated with risk of cervical cancer in a Chinese population. Gynecol Oncol 122: 33-37, 2011.

15. Wang J, Bi J, Liu X, et al: Hsa-miR-146a polymorphism (rs2910164) and cancer risk: a meta-analysis of 19 case-contro studies. Mol Biol Rep 39: 4571-4579, 2012.

16. Xu T, Zhu Y, Wei QK, et al: A functional polymorphism in the miR-146a gene is associated with the risk for hepatocellular carcinoma. Carcinogenesis 29: 2126-2131, 2008.

17. Ye Y, Wang KK, Gu J, et al: Genetic variations in microRNArelated genes are novel susceptibility loci for esophageal cancer risk. Cancer Prev Res (Phila): 460-469, 2008.

18. Srivastava K, Srivastava A and Mittal B: Common genetic variants in pre-microRNAs and risk of gallbladder cancer in North Indian population. J Hum Genet 55: 495-499, 2010

19. Guo H, Wang K, Xiong G, et al: A functional variant in microRNA-146a is associated with risk of esophageal squamous cell carcinoma in Chinese Han. Fam Cancer 9: 599-603, 2010.

20. Zeng Y, Sun QM, Liu NN, et al: Correlation between pre $m i R-146 a \mathrm{C} / \mathrm{G}$ polymorphism and gastric cancer risk in Chinese population. World J Gastroenterol 16: 3578-3583, 2010.

21. Min KT, Kim JW, Jeon YJ, et al: Association of the miR$146 a \mathrm{C}>\mathrm{G}, 149 \mathrm{C}>\mathrm{T}, 196 a 2 \mathrm{C}>\mathrm{T}$, and $499 \mathrm{~A}>\mathrm{G}$ polymorphisms with colorectal cancer in the Korean population. Mol Carcinog 51 (Suppl 1): E65-E73, 2012

22. Akkiz H, Bayram S, Bekar A, et al: No association of pre-microRNA-146a rs2910164 polymorphism and risk of hepatocellular carcinoma development in Turkish population: a case-control study. Gene 486: 104-109, 2011.

23. Hishida A, Matsuo K, Goto Y, et al: Combined effect of miR-146a rs2910164 G/C polymorphism and Toll-like receptor 4 $+3725 \mathrm{G} / \mathrm{C}$ polymorphism on the risk of severe gastric atrophy in Japanese. Dig Dis Sci 56: 1131-1137, 2011.

24. Okubo M, Tahara T, Shibata T, et al: Association between common genetic variants in pre-microRNAs and gastric cancer risk in Japanese population. Helicobacter 15: 524-531, 2010.

25. Zhang XW, Pan SD, Feng YL, et al: Relationship between genetic polymorphism in microRNAs precursor and genetic predisposition of hepatocellular carcinoma. Zhonghua Yu Fang Yi Xue Za Zhi 45: 239-243, 2011 (In Chinese).

26. Xiang Y, Fan S, Cao J, et al: Association of the microRNA-499 variants with susceptibility to hepatocellular carcinoma in a Chinese population. Mol Biol Rep 39: 7019-7023, 2012.

27. Kim WH, Min KT, Jeon YJ, et al: Association study of microRNA polymorphisms with hepatocellular carcinoma in Korean population. Gene 504: 92-97, 2012

28. Zhou F, Zhu H, Luo D, et al: A functional polymorphism in Pre-miR-146a is associated with susceptibility to gastric cancer in a Chinese population. DNA Cell Biol 31: 1290-1295, 2012

29. Ahn DH, Rah H, Choi YK, et al: Association of the miR-146a $\mathrm{C}>\mathrm{G}$, miR-149T $>\mathrm{C}$, miR-196a2T $>\mathrm{C}$, and miR-499A $>\mathrm{G}$ polymorphisms with gastric cancer risk and survival in the Korean population. Mol Carcinog 52 (Suppl 1): 39-51, 2013.

30. Hezova R, Kovarikova A, Bienertova-Vasku J, et al: Evaluation of SNPs in miR-196-a2, miR-27a and miR-146a as risk factors of colorectal cancer. World J Gastroenterol 18: 2827-2831, 2012.
31. Mihalache F, Hoblinger A, Acalovschi M, et al: A common variant in the precursor miR-146a sequence does not predispose to cholangiocarcinoma in a large European cohort. Hepatob Pancreat Dis Int 11: 412-417, 2012.

32. Zhou J, Lv R, Song X, et al: Association between two genetic variants in miRNA and primary liver cancer risk in the Chinese population. DNA Cell Biol 31: 524-530, 2012.

33. Song MY, Su HJ, Zhang L, et al: Genetic polymorphisms of $m i R-146 a$ and $m i R-27 a, H$. pylori infection, and risk of gastric lesions in a Chinese population. PLoS One 8: e61250, 2013.

34. Ma L, Zhu L, Gu D, et al: A genetic variant in miR-146a modifies colorectal cancer susceptibility in a Chinese population. Arch Toxicol 87: 825-833, 2013

35. Lv M, Dong W, Li L, et al: Association between genetic variants in pre-miRNA and colorectal cancer risk in a Chinese population. J Cancer Res Clin Oncol 139: 1405-1410, 2013.

36. Pavlakis E, Papaconstantinou I, Gazouli M, et al: MicroRNA gene polymorphisms in pancreatic cancer. Pancreatology 13: 273-278, 2013

37. Yuan Z, Zeng X, Yang D, et al: Effects of common polymorphism rs11614913 in Hsa-miR-196a2 on lung cancer risk. PLoS One 8: e61047, 2013

38. Liu J, Liu J, He Y, et al: Genetic variants in the microRNA machinery gene GEMIN4 are associated with risk of prostate cancer: a case-control study of the Chinese Han population. DNA Cell Biol 31: 1296-1302, 2012.

39. Luo J, Cai Q, Wang W, et al: A microRNA-7 binding site polymorphism in HOXB5 leads to differential gene expression in bladder cancer. PLoS One 7: e40127, 2012.

40. Zhou B, Wang K, Wang Y, et al: Common genetic polymorphisms in pre-microRNAs and risk of cervical squamous cell carcinoma. Mol Carcinog 50: 499-505, 2011.

41. Löfgren SE, Frostegård J, Truedsson L, et al: Genetic association of miRNA-146a with systemic lupus erythematosus in Europeans through decreased expression of the gene. Genes Immun 13: 268-274, 2012.

42. Zilahi E, Tarr T, Papp G, et al: Increased microRNA-146a/b, TRAF6 gene and decreased IRAK1 gene expressions in the peripheral mononuclear cells of patients with Sjögren's syndrome. Immunol Lett 141: 165-168, 2012.

43. Lu J, Kwan BC, Lai FM, et al: Glomerular and tubulointerstitial miR-638, miR-198 and miR-146a expression in lupus nephritis. Nephrology 17: 346-351, 2012.

44. Halkein J, Tabruyn SP, Ricke-Hoch M, et al: MicroRNA-146a is a therapeutic target and biomarker for peripartum cardiomyopathy. J Clin Invest 123: 2143-2154, 2013.

45. Fan KL, Zhang HF, Shen J, et al: Circulating microRNAs levels in Chinese heart failure patients caused by dilated cardiomyopathy. Indian Heart J 65: 12-16, 2013

46. Zidar N, Boštjančič E, Glavač D and Stajer D: MicroRNAs, innate immunity and ventricular rupture in human myocardial infarction. Dis Markers 31: 259-265, 2011.

47. Jazdzewski K, Murray EL, Franssila K, et al: Common SNP in pre-miR-146a decreases mature miR expression and predisposes to papillary thyroid carcinoma. Proc Natl Acad Sci USA 105: 7269-7274, 2008

48. Yamashita J, Iwakiri T, Fukushima S, et al: The rs 2910164 $\mathrm{G}>\mathrm{C}$ polymorphism in microRNA-146a is associated with the incidence of malignant melanoma. Melanoma Res 23: 13-20, 2013.

49. Orsós Z, Szanyi I, Csejtei A, et al: Association of pre-miR-146a rs2910164 polymorphism with the risk of head and neck cancer. Anticancer Res 33: 341-346, 2013. 